\title{
Handeln statt Warten: Ein mehrstufiger Ansatz zur Bewältigung des Klimaproblems
}

\author{
Elinor Ostrom
}

\section{Erratum zu: Leviathan 39 (2), 267-278 \\ DOI: 10.1007/s11578-011-0114-1}

Aufgrund unglücklicher Umstände wurde der Essay von Elinor Ostrom in Heft 2/2011 in einer fehlerhaften Fassung publiziert. Deshalb folgt hier nun, mit einer Entschuldigung an die Leserschaft, die zitierbare Fassung.

\begin{abstract}
Zusammenfassung: Die Klimaproblematik ist sehr komplex und auf der internationalen politischen Agenda immer noch relativ neu. Daher ist es nicht sinnvoll, auf den Abschluss eines globalen Abkommens zu warten. Es wäre besser, einen mehrstufigen Lösungsansatz zu verfolgen, der auf der lokalen Ebene ansetzt, Vorteile klimapolitischer Anstrengungen auf mehreren Ebenen maximiert und einen Prozess des Experimentierens und Lernens über Klimapolitik initiiert. Die konventionelle Theorie kollektiven Handelns sagt uns, dass die effiziente Nutzung von Ressourcen nur durch zwei Maßnahmen möglich ist: Privatisierung oder Verstaatlichung. Diese Ansicht behindert Fortschritte. Um dem Klimawandel langfristig erfolgreich zu begegnen, müssen sich alltägliche Verhaltensmuster von Einzelpersonen, Familien, Firmen, Gemeinden und Regierungen auf verschiedenen Ebenen ändern - insbesondere in den entwickelten Ländern.
\end{abstract}

Schlüsselwörter: Klimapolitik · Öffentliche Güter · Mehrstufiger Ansatz

(C) VS Verlag für Sozialwissenschaften 2011

Die Online-Version des Originalartikels können Sie unter doi:10.1007/s11578-011-0114-1 finden

Dieser Beitrag basiert auf der Climate Lecture ,Who affects climate change', die Elinor Ostrom am 22. Juli 2010 an der Technischen Universität Berlin gehalten hat. Der Einführungsvortrag zu dieser Climate Lecture wurde von Ottmar Edenhofer gehalten und ist bereits im Leviathan (Heft 2/2011) erschienen.

Übersetzung: Steffen Brunner und Christian Flachsland

Prof. Dr. E. Ostrom $(\bowtie)$

Workshop in Political Theory and Policy Analysis, Indiana University,

513 North Park Avenue, Bloomington, IN, 47408-3895, USA

E-Mail: ostrom@indiana.edu 


\title{
A multi-scale approach to coping with climate change and other collective action problems
}

\begin{abstract}
Climate change is a complex problem and still relatively new on the international agenda. Waiting for effective climate policies to be established at the global level is unreasonable and wastes valuable time. It would be better to adopt a multi-scale approach starting at the local level, maximizing benefits at varying levels and encouraging experimentation and learning from diverse policies. Conventional wisdom tells us that there are only two options for dealing with resource management: either privatization or management by the state. This view is hindering progress. Successfully addressing climate change in the long run requires substantial changes at multiple levels in the day-to-day activities of individuals, families, firms, communities, and governments especially those in the more developed world.
\end{abstract}

Keywords: Climate policy $\cdot$ Public goods $\cdot$ Multi-scale approach

\section{Herausforderung Klimawandel}

Kein Land der Welt - auch kein reiches - kann das Klimaproblem alleine lösen. Gleichzeitig kann nicht darauf gewartet werden, dass die internationalen Verhandlungen eine globale Lösung liefern. Wie die Verhandlungen in Kopenhagen im Dezember 2009 gezeigt haben, herrscht zwischen den großen Staaten erhebliche Uneinigkeit über den erforderlichen Umfang von Emissionsreduktionen (Matthews und Caldeira 2008).

Wichtige Debatten werden über Schlüsselfragen zur Einrichtung effizienter und fairer Mechanismen auf globaler Ebene geführt. Wer ist für die hohen $\mathrm{CO}_{2}$ Konzentrationen in der Atmosphäre verantwortlich (Botsen et al. 2008; Dellink et al. 2009)? Wer sollte die Hauptlast der Kosten für Lösungsansätze tragen (Baer et al. 2000; Najam et al. 2003; Posner und Sunstein 2008)? Und können die „Patentrezepte“, die zur Reduktion von $\mathrm{CO}_{2}$ Emissionen vorgeschlagen wurden, auch zur Lösung anderer Umweltprobleme beitragen? Eine andere wichtige Frage ist, ob die Abholzung von Wäldern mit der Folge der Freisetzung von $\mathrm{CO}_{2}$-Emissionen und Veränderungen in regionalen Wasserkreisläufen (verminderte Wolkenbildung) den Klimawandel in der Nettobilanz verstärkt, oder ob der kühlende Effekt einer helleren Erdoberfläche - insbesondere in Wäldern der nördlichen Hemisphäre - dominiert (Bala et al. 2007). Zudem ist es umstritten, ob Zahlungen für ökosystemare Dienstleistungen tatsächlich die beiden Ziele der $\mathrm{CO}_{2}$ - Einlagerung in Biomasse und die Erhaltung der Artenvielfalt gleichzeitig erreichen können (Nelson et al. 2008).

Angesichts des nun schon Jahrzehnte währenden Scheiterns der Verhandlungen über ein effizientes, gerechtes und durchsetzbares Abkommen über Emissionsreduktionen auf globaler Ebene könnte ein fortgesetztes Warten dazu führen, dass eine tragische Entwicklung nicht mehr gestoppt werden kann. Statt sich auf die zu erwartenden Risiken zu fokussieren, wurde zu viel Wert auf das Erreichen eines bestimmten Reduktionsziels gelegt. Die Reduktion von Emissionen - jetzt - ist dringlicher als ein internationales Abkommen über Reduktionsverpflichtungen, das noch einige Zeit auf sich warten lassen könnte. Wir befinden uns nicht - wie bei einigen erneuerbaren Ressourcen - in einer Situation, in der durch zusätzliche Nutzung der Atmosphäre unterhalb eines kritischen Schwellenwertes kein größerer Schaden entsteht. In Anbetracht der wichtigen Rolle tech- 
nologischer Innovationen bei der Lösung des Klimaproblems würden wir ohne den Start technologischer und institutioneller Anstrengungen auf mehreren Ebenen nicht einmal beginnen zu lernen, welches Bündel an Maßnahmen für die Abwehr der langfristigen Bedrohung durch einen massiven Klimawandel letztlich am effektivsten ist.

Zusätzlich zum Problem des zu langen Abwartens müssen global verhandelte Lösungen in jedem Fall durch Anstrengungen auf nationaler, regionaler und lokaler Ebene ergänzt werden, um erfolgreich zu sein. Während die Konzentration von $\mathrm{CO}_{2}$ und anderen Treibhausgasen weltweit relativ gleich verteilt ist, sind die Auswirkungen des Klimawandels von Region zu Region sehr verschieden. Sie werden durch geographische, ökologische und ökonomische Rahmenbedingungen, bereits geleistete Anpassungsmaßnahmen und vergangene Investitionen bestimmt. Jene, die am meisten unter den Auswirkungen des Klimawandels leiden werden, sind auf internationaler politischer Ebene nur unzureichend repräsentiert und möglicherweise nicht in der Lage, günstige Reduktionsmaßnahmen für Treibhausgasemissionen oder Möglichkeiten zur Anpassung an die Vielzahl der sie bedrohenden Gefahren an andere zu vermitteln (Agrawal 2008).

Viele Auswirkungen des Klimawandels wirken zwar global, die Ursachen des Klimawandels aber liegen auf einer niedrigeren Ebene - der bekannte Slogan ,Think globally, act locally“ drückt das alle Menschen auf diesem Planeten betreffende Problem sehr gut aus. Um das Klimaproblem langfristig lösen zu können, müssen alltägliche Aktivitäten von Einzelpersonen, Familien, Firmen, Gemeinden und Regierungen auf verschiedenen Ebenen - insbesondere in den industrialisierten Ländern - wesentlich verändert werden. Selbst Gestaltungsmöglichkeiten auf der Familienebene können einen wesentlichen Unterschied ausmachen (Dietz et al. 2009; Gardner und Stern 2008; Vandenbergh et al. 2008). Wenn Familien ihr Verhalten bei der Isolierung von Häusern, der Bildung von Fahrgemeinschaften und dem Kauf sparsamer Autos ändern, können sie im Kleinen mit ihren Aktionen eine Reduzierung ihrer Treibhausgasemissionen und ihres Energieverbrauchs um rund $30 \%$ bewirken (Vandenbergh und Steinemann 2007).

Es sind Milliarden von Akteuren, die die globale Atmosphäre beeinflussen. Jeder wird von einer Reduzierung der Treibhausgasemissionen profitieren, egal ob er zu dieser Anstrengung beiträgt oder nicht. Klimaschutz erfordert also die Sicherstellung eines öffentlichen Gutes, und die Produktion öffentlicher Güter beinhaltet das klassische Dilemma kollektiven Handelns. Es ist das vielleicht größte Dilemma, dem die Menschheit je wissentlich gegenüberstand. Die klassische Theorie des kollektiven Handelns sagt voraus, dass niemand sein Verhalten ändern und den Energieverbrauch senken wird, solange nicht eine übergeordnete Instanz durchsetzbare Regeln auferlegt, die die Anreize aller Beteiligten verändert. Aus diesem Grund fordern viele Forscher institutionelle Veränderungen auf globaler Ebene (Stavins 1997; Miller 2004; Wiener 2007). Angesichts der Diagnose, dass globale Probleme nur global ,gelöst“ werden können, sollte die Klimaforschung folgende Fragen näher untersuchen:

1. Ist die herkömmliche Theorie des kollektiven Handelns tatsächlich die beste Theorie zur Analyse der Möglichkeiten der Vermeidung der Bedrohungen durch einen massiven Klimawandel?

2. Wenn dies nicht der Fall ist, welche wesentlichen Annahmen müssen sich hinsichtlich (a) der grundlegenden Theorie und (b) der Größenordnung des Vermeidungspotenzials sub-globaler Anstrengungen ändern? 
3. Stiften lokale Emissionsreduktionen weltweiten Nutzen? Können sie auch auf mehreren Ebenen vorteilhaft sein?

4. Gibt es derzeit Vermeidungs- und Anpassungsanstrengungen auf sub-globaler Ebene?

5. Sind große Regierungsapparate besser für die Bewältigung großskaliger kollektiver Handlungsprobleme geeignet?

Diese Fragen werden im Folgenden der Reihe nach diskutiert.

\section{Die konventionelle Theorie des kollektiven Handelns}

Der Begriff „soziales Dilemma“ bezeichnet eine Situation, in der unkoordinierte Entscheidungen von eigeninteressierten Individuen zu langfristig suboptimalen Ergebnissen für den Einzelnen und für Andere führen. Das sozial optimale Ergebnis könnte erreicht werden, wenn die meisten Beteiligten „kooperieren“ würden. Doch angesichts der Tatsache, dass alle erst einmal abwarten, verspürt niemand einen Anreiz, sein eigenes Verhalten zu ändern (Costanza 1987; Sandler 2004).

Im Fall des Klimawandels profitieren wir alle von der Reduktion von Treibhausgasemissionen. Das gemeinsame Ziel ist die Verringerung der Bedrohungen durch den massiven Klimawandel - steigende Meeresspiegel, zunehmende Klimavariabilität und viele andere globale Kosten. Die traditionelle Theorie des kollektiven Handelns sagt voraus, dass ohne von außen durchgesetzte Regulierung mögliche Vorteile der Kooperation nicht realisiert werden können. In der herkömmlichen Theorie gibt es keine „,selbstorganisierten Gruppen“, die ihre eigene Politik entwickeln können, um ein öffentliches Gut oder eine Allmende zu bewirtschaften. Die Anwendbarkeit der konventionellen Theorie auf das Klimaproblem wurde von vielen Wissenschaftlern als so selbstverständlich erachtet, dass nur wenige hinterfragt haben, ob dies tatsächlich die beste theoretische Grundlage ist, um echte Fortschritte bei der Verringerung von Treibhausgasemissionen zu erreichen.

Es gibt zwei gute Gründe, daran zu zweifeln. Der erste ist die schwache empirische Bestätigung der Voraussagen dieser Theorie in kleinen bis mittelgroßen ökologischen Dilemmasituationen. Der zweite ist die Existenz von mehreren Externalitäten auf kleinen, mittleren und großen Ebenen innerhalb der globalen Klimaexternalität, die bisher das Hauptanliegen der akademischen und politischen Literatur darstellt.

\section{Die schwache empirische Basis}

In einer breit angelegten Meta-Analyse prüfen Potetee et al. (2010) eine Reihe von auf verschiedenen Methoden basierenden Studien daraufhin, ob sie die Voraussagen der konventionellen Theorie kollektiven Handelns empirisch belegen. Während in der empirischen Forschung tatsächlich viele Fälle des Trittbrettfahrens beobachtet werden, gibt es auch eine überraschend große Zahl von Fällen, in denen Individuen kollektive Handlungsprobleme durch Kooperation lösen. Im Gegensatz zur herkömmlichen Theorie organisieren sich in diesen Situationen Gruppen auf kleiner bis mittlerer Ebene selbständig, 
um Lösungsansätze für ihre sozialen Dilemmata zu entwickeln (Ostrom 1992, 2001; Ostrom et al. 1994; NRC 2002; Dietz et al. 2003).

Dabei wird die Wahrscheinlichkeit effektiver Selbstorganisation zur Überwindung kollektiver Handlungsprobleme durch eine ganze Reihe von Variablen erhöht. Die wichtigsten sind (1) Verfügbarkeit zuverlässiger Informationen über die kurz- und langfristigen Kosten und den Nutzen der Maßnahmen, (2) die beteiligten Personen betrachten die Ressource als wichtig für ihre eigenen Ziele und haben einen langfristigen Zeithorizont, (3) der Aufbau einer Reputation als vertrauenswürdiger Transaktionspartner ist den Beteiligten wichtig, (4) die Akteure können wenigstens mit einigen der anderen Beteiligten kommunizieren, (5) Mechanismen der informellen Überwachung und Sanktionierung sind durchführbar und werden als angemessen erachtet und (6) Sozialkapital und Führungsbereitschaft sind durch die erfolgreiche Lösung früherer gemeinsamer Probleme vorhanden.

Die Situationsstrukturen zur Verbesserung der Chancen auf Zusammenarbeit können nicht allgemein und abstrakt bestimmt werden. Vielmehr werden die Chancen auf Erfolg durch die jeweilige Kombination der spezifischen Besonderheiten jeder Dilemmasituation beeinflusst. Entscheidend ist, dass eine Kombination struktureller Merkmale vorliegt, die das Vertrauen der Beteiligten untereinander stärkt und ihre Bereitschaft fördert, eine gemeinsam beschlossene Maßnahme trotz kurzfristiger individueller Kosten durchzuführen. Selbstorganisation angesichts eines sozialen Dilemmas kann nur funktionieren, wenn die Handelnden davon längerfristigen Nutzen sowohl für sich selbst als auch für andere erwarten können und wenn die Beteiligten glauben, dass sich jeder an die Vereinbarung hält.

Das Problem des kollektiven Handelns verschwindet nicht, sobald eine Regierung entsprechende politische Maßnahmen beschlossen hat. Auch staatliche Maßnahmen sind auf die freiwillige Kooperation von Bürgern angewiesen. Wenn Bürger eine Politik befürworten und meinen, dass sie entsprechend handeln sollten, und wenn sie erkennen, dass diese staatliche Politik auch effektiv und gerecht durchgesetzt wird, fallen die Kosten für die Durchsetzung viel niedriger aus, als wenn die Bürger versuchen, sich der Politik zu entziehen. Das Vertrauen, dass Regierungsbeamte objektiv, effektiv und gerecht sind, ist für das Funktionieren politischer Regulierung wichtiger als staatlicher Zwang (Rothstein 1998, 2005).

\section{Das Vorhandensein mehrerer Externalitäten}

Es ist natürlich viel einfacher, Lösungen für Probleme kollektiven Handelns für kleinere Allmenden zu finden, als für globale Gemeinschaftsgüter. Viele Analysen des Klimaproblems, die „Lösungen“ auf internationaler Ebene empfehlen, die dann von nationalen Regierungen umgesetzt werden müssen, beruhen auf der Befürchtung, dass ohne einen globalen Lösungsansatz letztlich überhaupt nichts passieren wird. Die dritte der oben angesprochenen Fragen lautete: „Stiften lokale Emissionsreduktionen nur weltweiten Nutzen oder können sie auf mehreren Ebenen vorteilhaft sein?“ Entscheidungen innerhalb von Familien über die Wahl der Transportmittel, die Isolierung des Hauses und über Investitionen, die den häuslichen Stromverbrauch verringern, haben kleine (aber in der 
Summe wichtige) Auswirkungen auf die globale Atmosphäre. Entscheidungen in Unternehmen sind ähnlich wichtig, weil Gebäude in den Vereinigten Staaten mehr als $70 \%$ der Elektrizität verbrauchen und durch ihren Betrieb fast $40 \%$ der Treibhausgasemissionen entstehen (Fuller et al. 2009).

Wenn regionale Stromnetze die Nutzung von in Haushalten generiertem Solarstrom für den häuslichen Gebrauch oder die Einspeisung in das Netz erlauben, können auf diesem Weg Energiekosten und Treibhausgasemissionen reduziert werden. Investitionen in bessere Abfallentsorgungsanlagen bringen lokalen Nutzen und reduzieren die globalen Emissionen. Maßnahmen zur Bekämpfung der Luftverschmutzung in Großstädten verringern den weltweiten Energieverbrauch und die lokalen Emissionen und Nachteile der Verschmutzung durch Luftpartikeln. Die Reduktion von Subventionen für emissionsintensive Industrien ist zwar für alle Regierungen ein schwieriges Unterfangen, aber solche Maßnahmen können gleichzeitig Kosten sparen und die Umwelt schützen.

\section{Können große Regierungsapparate kollektive Handlungsprobleme besser lösen?}

Viele Analysen kommen zu dem Schluss, dass der Klimawandel globale Lösungsansätze erfordert, weil lokale und regionale Anstrengungen nicht ausreichen werden. Allerdings gibt es nur wenige Analysen der Probleme, die große Regierungsapparate bei der Entwicklung effektiver Ressourcenpolitik haben können. Bevor wir die Entscheidung treffen, dass die globale Ebene die einzig richtige Regulierungsebene für den Klimaschutz ist, sollten wir zumindest bisherige Erfahrungen mit der zentralisierten Politik großer Regierungsapparate zur Lösung kollektiver Handlungsprobleme in Augenschein nehmen. Die Annahme, dass lokale Akteure zur Bearbeitung öffentlicher Probleme nicht in der Lage sind, hat immer wieder dazu geführt, dass lokale öffentliche Dienstleistungen auf höhere Regulierungsebenen verlagert wurden - allerdings ohne dass diese Ebenen mit den zur Aufgabenerfüllung nötigen Ressourcen ausgestattet wurden.

Diese gegenwärtige Praxis der Verlagerung der gesamten Verantwortung für die Bereitstellung lokaler öffentlicher Güter und Gemeingüter an regionale, nationale oder internationale Instanzen hemmt die Möglichkeiten lokaler Beamter und Bürger, lokale Probleme selbst zu lösen. Aufgrund des Unvermögens der internationalen Organisationen, die internationalen Fischbestände effektiv zu bewirtschaften, hat die Seerechtskonferenz der Vereinten Nationen etwa ein Drittel der ,internationalen Gewässer“، Nationalstaaten zugeordnet (United Nations 1982). Die 200 Seemeilen-Zone entlang der Küsten wurde als nationalstaatlich kontrollierte Ausschließliche Wirtschaftszone (AWZ) definiert, um Küstenfischbestände vor der Übernutzung zu schützen. Doch anstatt die Überfischung zu reduzieren, subventionierten viele Staaten den Ausbau ihrer Fischereiflotten und verschärften damit die Übernutzungsprobleme zusätzlich (Walters 1986). Die von den Staaten benutzten Computermodelle waren sehr grob und produzierten ungenaue Bestandsschätzungen (Wilson 2002).

Das für Fischerei und Meere zuständige Ministerium in Kanada etwa entwickelte ein von Wissenschaftlern im Nachhinein als fehlerhaft eingeschätztes Modell der Bestandsregeneration regionaler Dorschbestände (Harris 1990). In den späten 1980er Jahren bezweifelten lokale Fischer in Neufundland die Korrektheit der Modellergebnisse und 
prognostizierten einen baldigen Zusammenbruch der Bestände. Die kanadische Regierung weigerte sich allerdings darauf einzugehen und versicherte allen Zweiflern, dass ihr Modell richtig sei. Im Jahr 1992 jedoch brach der Dorschbestand zusammen und die kanadische Regierung erklärte ein Fischfangmoratorium für die kanadischen Gewässer. Dies erzeugte sehr hohe Kosten in den von der Fischerei lebenden Ortschaften, die den Fischbestand vorher relativ effektiv verwaltet hatten (Finlayson 1994; Finlayson und McCay 1998). Das Fischereibeispiel zeigt, dass große Regierungsapparate in der Verwaltung großskaliger - in diesem Beispiel allerdings nicht globaler - Ressourcen nicht notwendigerweise bessere wissenschaftliche Urteile und Entscheidungen fällen.

Der Hinweis auf die Probleme größerer Regierungsapparate soll nicht die Notwendigkeit einer globalen Klimapolitik in Frage stellen. Die Absicht ist vielmehr, den engen Fokus auf globale Lösungen als primäre Strategie zur Bewältigung des Klimaproblems zu erweitern. Die Erforschung der Rolle von Institutionen in der Umweltpolitik hat wiederholt gezeigt, dass es kreative, effektive und effiziente Politik, aber auch katastrophale Fehler auf allen Regulierungsebenen gegeben hat. Die Beschäftigung mit der Komplexität von Umweltproblemen kann zu „,negativem Lernen“ bei Wissenschaftlern und politischen Entscheidungsträgern führen (Oppenheimer et al. 2008). Und die Fokussierung auf die eine einzig ,richtige Lösung“ wird eher zur Verschärfung des Problems führen als zu seiner Lösung (Pritchett und Woolcock 2003). Wir müssen daher anerkennen, dass Politikmaßnahmen im Zusammenhang mit komplexen ökologischen Prozessen eine große Herausforderung darstellen und dass das Vertrauen auf eine einzige Regulierungsebene naiv ist.

\section{Was lernen wir daraus?}

Die Diskussion über die Vorteile lokaler und nationaler gegenüber globalen Anstrengungen um Emissionsreduktionen produziert eine Menge heißer Luft, aber nicht unbedingt bessere Lösungen. Wir müssen eine Reihe von Tatsachen anerkennen. (1) Die Ursachen des Klimawandels sind komplex, (2) in einer sich schnell verändernden Welt ist es schwierig, Ursachen und Wirkungen zu verstehen, (3) es gibt eine Reihe von Politikmaßnahmen zur Reduktion von Emissionen, von denen einige allerdings zu opportunistischem Verhalten und der Erschleichung von Unterstützungszahlungen ohne nennenswerte Reduktionen führen können, oder, noch schlimmer, einen Anstieg der Emissionen verursachen, (4) Experimente mit Politikinstrumenten könnten möglicherweise dann in größerem Ausmaß finanziell unterstützt werden, wenn Geldmittel zur Überwachung und Bewertung von Kosten und Nutzen solcher Projekte bereitgestellt werden, und (5) politische Maßnahmen können auf jeder Ebene scheitern, doch ohne Versuch und Irrtum lernt man nicht.

In Anbetracht der Komplexität des Problems und des noch jungen wissenschaftlichen Konsensus über die multiplen, lokalen Ursachen des Klimawandels ist das Warten auf effektive Politik auf globaler Ebene unvernünftig. Statt immer nur auf die Verhandlungen auf globaler Ebene zu starren, wäre ein mehrstufiger Ansatz zur Bearbeitung des Problems des Klimawandels effektiver und würde einen Prozess des Experimentierens und Lernens fördern. Wie aber könnte ein mehrstufiger Ansatz aussehen? 


\section{Ein mehrstufiger Lösungsansatz}

Wie oben angesprochen, haben Emissionsreduktionen auf mehreren Ebenen positive Nebeneffekte. Mögliche Vorteile bestehen sogar auf der Ebene von Haushalten: Der Verzicht auf das Auto zugunsten eines Fahrrads für den Weg zur Arbeit verbessert die körperliche Kondition der Menschen. Die verbesserte Architektur von Gebäuden, Sanierungen, die Installierung von Solarzellen und viele andere Maßnahmen senken Heiz- und Stromkosten und können damit für Familien und Unternehmen langfristig profitabel sein.

Darüber hinaus hat die umfangreiche empirische Forschung über kollektive Handlungsprobleme wiederholt gezeigt, dass Vertrauen und Reziprozität zwischen den Beteiligten zentrale Voraussetzungen für erfolgreiches kollektives Handeln sind. Würde Klimapolitik allein auf globaler Ebene implementiert, dann wäre es für Bürger und Firmen ausgesprochen schwierig, darauf zu vertrauen, dass in anderen Erdteilen ähnliche Anstrengungen unternommen werden wie bei ihnen ,zu Hause“. Wenn Beteiligte befürchten müssen, dass sie als die „Gelackmeierten“ dastehen werden, wenn sie selbst kostenintensive Maßnahmen ergreifen und andere als Trittbrettfahrer auftreten, werden sie ihre Ressourcen auf die Vortäuschung von Emissionsreduktionen verwenden. Ein Schlüsselproblem ist daher die Überwachung von Maßnahmen (Monitoring).

\section{Verschiedene Monitoringstrategien}

Der Rückgriff auf bereits bestehendes Vertrauen und bestehende Reziprozität zwischen Akteuren in einer kollektiven Dilemmasituation ist wenig erfolgversprechend. Das gilt besonders dann, wenn neue, bisher unbekannte Probleme auftreten. Um ein nachhaltig funktionierendes System kollektiven Handelns zu errichten, müssen die entsprechenden Regeln auch tatsächlich durchgesetzt werden. Diese Frage wird bei Reformanstrengungen häufig vernachlässigt. In diesem Zusammenhang ist unsere Forschung über Ressourcenregime relevant (Gibson et al. 2000; Ostrom und Nagendra 2006). Bei den Bemühungen um die Bereitstellung eines anderen kollektiven Gutes, der Artenvielfalt, wurden zahlreiche Schutzgebiete entworfen, die letztlich nur auf dem Papier der weit entfernten Geldgeber existierten, während die Gebiete in Wahrheit durch illegale Rodung zerstört wurden. Viele stimmen der Forderung zu, dass die Durchsetzung von Regeln für eine nachhaltige Ressourcenbewirtschaftung ganz zentral ist. Allerdings herrscht Uneinigkeit über die Frage, wer die Regeleinhaltung überwachen sollte (Bruner et al. 2001; Wells). Einfach ein paar Aufseher anzustellen, die weder die Gegend noch deren Bewohner kennen, hat sich in der Vergangenheit als wenig erfolgreich erwiesen.

Die meisten langlebigen Ressourcenregime wählen ihre Aufseher selbst aus. Diese sind gegenüber den Nutzern rechenschaftspflichtig, oder sind selbst Nutzer und haben die Aufgabe, den Zustand der Ressource und die Nutzungsaktivitäten zu beobachten. Ein auf lokalem Monitoring beruhendes Ressourcenregime muss zur Ahndung von Regelverstößen nicht ausschließlich auf lokale Normen zurückgreifen. Die Gemeinschaft kann selbst ein offizielles Amt einrichten. In einigen Systemen rotiert das Amt des Aufsehers, so dass 
jeder Nutzer diese Rolle reihum übernimmt. In anderen Systemen zahlen alle Beteiligten einen Beitrag und engagieren gemeinsam Aufseher, die das Monitoring übernehmen.

In einer vom Forschungsnetzwerk International Forestry Resources and Institutions (IFRI) durchgeführten Studie über den Zustand von Wäldern, die 178 Benutzergruppen in zwölf verschiedenen Ländern umfasst, haben Gibson et al. (2005) festgestellt, dass die Qualität der lokalen Monitoringsysteme stark variiert. Eine der untersuchten Variablen war die Häufigkeit, mit der lokale Gruppen Regelverstöße überwachen und sanktionieren. Wir untersuchten den Einfluss dieser Variablen auf die Einschätzung des Waldzustands durch die Nutzer (und einen Förster). Wir untersuchten zudem die Rolle von Sozialkapital, die Abhängigkeit der Gruppe von Waldressourcen, und ob die Gruppe formal organisiert war oder nicht. Das Ergebnis der Analyse war, dass regelmäßiges Kontrollieren durch eine lokale Gruppe für den gesunden Zustand des Waldes am wichtigsten war. Unabhängig von der Qualität des Sozialkapitals, der Abhängigkeit von Waldressourcen und formaler Organisation erwiesen sich regelmäßige Überwachung und Sanktionierung als bester Weg zur Verbesserung des Waldzustands (Hayes und Ostrom 2005).

Einige lokale Stromversorger in den USA versuchen nun, durch die Entwicklung lokaler Monitoringsysteme und die Weitergabe der Ergebnisse auf Kundenrechnungen den Energieverbrauch zu senken. Die Stadtwerke von Sacramento beispielsweise haben bereits verschiedene Methoden erprobt, darunter Rabatte für energiesparende Geräte. Sie sind allerdings vor kurzem auf ein noch erfolgreicheres Modell gestoßen. Im April 2008 begannen sie damit, an 35000 zufällig ausgewählte Kunden Daten über ihren Energieverbrauch zu versenden, anhand derer sie ihren Verbrauch mit 100 Häusern ähnlicher Größe und gleichen Brennstoffen vergleichen konnten. Die Kunden konnten sich auch mit 20 Nachbarn vergleichen, die besonders effizient im Energiesparen waren und erhielten ein oder zwei „Smileys“ als persönliche Auszeichnungen auf ihrer monatlichen Abrechnung, je nachdem wie groß ihre Einsparungen waren. Nach sechs Monaten fanden die Stadtwerke, „dass Kunden, die den personalisierten Verbrauchsbericht erhielten, ihren Energieverbrauch um 2\% reduzierten im Vergleich mit jenen, die nur eine Standardabrechnung erhielten“ (Kaufmann 2009). Verschiedene Formen des Wettbewerbs zwischen privaten Haushalten und Gruppen sowie eine Rückmeldung darüber, wer am besten und mit welchen Mitteln den Energieverbrauch senkt, sind Strategien zur Verringerung von Emissionen, die nun zunehmend von Universitäten, kleineren Städten und Versorgungsfirmen im ganzen Land übernommen werden.

Neuere psychologische Studien zeigen, dass ein sozialer Rahmen bei der Nutzung von Ressourcen Handlungsweisen entscheidend beeinflusst. Schultz et al. (2007) und Mumford (2007) berichten, dass Nachrichten mit Bezügen zum sozialen Umfeld auf wirkungsvollere Weise Verhaltensänderungen auslösen als Nachrichten, die Sachinformationen betonen. Ein Beispiel: Der Hinweis „, $\mathrm{x} \%$ unserer Hotelgäste verwenden ihre Handtücher wieder" ist für Verhaltensänderungen effektiver als eine Aussage über die Menge an Wasser, die durch Wiederverwendung gespart werden könnte. 


\section{Komplexe mehrstufige Systeme zur Bewältigung komplexer mehrstufiger Probleme}

Da die Anerkennung des Klimawandels als Bedrohung für viele Bürger und Entscheidungsträger noch relativ neu ist, und da immer noch über die Verursacher und die für die Lösung Verantwortlichen diskutiert wird, kann zwar nicht erwartet werden, dass in der unmittelbaren Zukunft ein effektives, mehrstufiges Politikregime implementiert wird. Aber angesichts der langsamen Fortschritte und der Konflikte, die ein globaler Lösungsansatz mit sich bringt, sind gerade Initiativen zur Erkundung des Potentials effektiver Senkungen des Energieverbrauchs auf mehreren Ebenen ein wichtiger Schritt voran.

Eine der wichtigsten Strategien zur $\mathrm{CO}_{2}$-Reduktion ist zudem der wirksamere Schutz von Ökosystemen, die Kohlenstoff einlagern. Die Entwicklung wirksamer und anpassungsfähiger Programme erfordert jedoch die Wahl geeigneter Flächen und Investitionen in die Entwicklung von Schutzgebieten und technologische Infrastruktur (Michel 2009). So sind etwa erhebliche Investitionen in die wissenschaftliche Modellentwicklung erforderlich (Nelson et al. 2009). Glücklicherweise stehen nun dank der jüngsten Durchbrüche bei der Entwicklung geographischer Informationssysteme und vertiefter Kenntnisse über die relevanten biophysikalischen Prozesse bei der Erstellung dynamischer Ökosystemkarten die nötigen Werkzeuge bereit, um ein sorgfältigeres Management von Ökosystemen zu gewährleisten (Daily et al. 2009). Die Skala dieser Modelle muss jedoch der jeweiligen Regulierungsebene angemessen sein, damit die sinnvollsten politischen Maßnahmen zur Kohlenstoffeinlagerung identifiziert werden können.

Angesichts der Komplexität und der sich wandelnden Probleme bei der Bearbeitung des Klimawandels gibt es keine „optimalen“ Lösungen zur Erreichung ambitionierter Emissionsreduktionen. Eine deutliche Verringerung der Emissionen ist aber auf jeden Fall nötig. Der Vorteil eines mehrstufigen Ansatzes ist, dass er experimentelle Initiativen auf mehreren Ebenen erlaubt, und dass er die Entwicklung von Methoden fördert, die die Vor- und Nachteile bestimmter Strategien vergleichbar machen. Eine starke Einbindung der lokalen Ebene bei der Suche nach Möglichkeiten zur Verringerung individueller Emissionen ist ein wichtiges Element der Vorsorge gegen den Klimawandel. Der Aufbau solcher Formen, das Einbinden der örtlichen Kräfte und das nötige Vertrauen, dass auch andere Verantwortung übernehmen, kann effektiver in kleinen bis mittleren Regulierungseinheiten sein, wenn sie mit Informationsnetzwerken und Monitoringsystemen auf mehreren Ebenen miteinander verbunden sind.

Danksagung: Dieser Artikel erschien zuerst als „A multi-scale approach to coping with climate change and other collective action problems" in The Solutions Journal 1, 2 (2010). Ich danke Tom Dietz für Kommentare und Christina Asquith für die Bearbeitung meines Beitrags.

\section{Literatur}

Agrawal, A. 2008. The role of local institutions in adaptation to climate change. Report submitted to the World Bank, Washington, DC.

Baer, P., et al. 2000. Equity and greenhouse gas responsibility. Science 289:2287. 
Bala, G., et al. 2007. Combined climate and carbon-cycle effects of large-scale deforestation. Proceedings of the National Academy of Sciences 104 (16): 6550-6555.

Botsen, W. J. W., J. M. Gowdy, und J. C. J. M. Van Den Bergh. 2008. Cumulative $\mathrm{CO}_{2}$ emissions: Shifting international responsibilities for climate debt. Climate Policy 8:569-576.

Bruner, A., R. Gullison, R. Rice, et al. 2001. Effectiveness of parks in protecting tropical biodiversity. Science 291 (5501): 125-129.

Costanza, R. 1987. Social traps and environmental policy. BioScience 37:407-412.

Daily, G. C., et al. 2009. Ecosystem services in decision making: Time to deliver. Frontier in Ecology and the Environment 7 (1): 21-28.

Dellink, R., et al. 2009. Sharing the burden of adaptation financing. Amsterdam: Institute for Environmental Studies Newsletter (January).

Dietz, T., E. Ostrom, und P. Stern. 2003. The struggle to govern the commons. Science 302 (5652): 1907-1912.

Dietz, T., G. T. Gardner, J. Gilligan, et al. 2009. The behavioral wedge: Household actions can rapidly reduce U.S. carbon emissions. Proceedings of the National Academy of Sciences 106:18452-18456.

Finlayson, A. C. 1994. Fishing for truth: A sociological analysis of Northern cod stock assessment from 1977-1990. St. Johns: Memorial University of Newfoundland, Newfoundland Institute of Social and Economic Research.

Finlayson, A. C., B. J. McCay. 1998. Crossing the threshold of ecosystem resilience: The commercial extension of Northern cod. In Linking social and ecological systems: Management practices and social mechanisms for building resilience, Hrsg. Fikret Berkes und Carl Folke, 311-338. Cambridge: Cambridge University Press.

Fuller, M. C., S. C. Portis, und D. Kammen. 2009. Toward a low-carbon economy: Municipal financing for energy efficiency and solar power. Environment 51 (1): 22-32.

Gardner, G. T., und P. C. Stern. 2008. The short list: The most effective actions U.S. households can take to curb climate change. Environment 50:13-24.

Gibson, C., M. McKean, und E. Ostrom, Hrsg. 2000. People and forests: Communities, institutions, and governance. Cambridge: MIT Press.

Gibson, C., J. Williams, und E. Ostrom. 2005. Local enforcement and better forests. World Development 33 (2): 273-284.

Harris, L. 1990. Independent review of the state of the Northern Cod Stock. Prepared for the Honourable Thomas Siddon, Minister of Fisheries. Ottawa: Communication Directorate, Department of Fisheries and Oceans.

Hayes, T., und E. Ostrom. 2005. Conserving the world's forests: Are protected areas the only way? Indiana Law Review 38 (3): 595-617.

Kaufman, L. A. 2009. Desire to keep up with neighbors is spurring conservation. New York Times, 30. Januar.

Matthews, H. D., und K. Caldeira. 2008. Stabilizing climate requires near zero emissions. Geophysical Research Letters 35:1-5.

Michel, D. 2009. Foxes, hedgehogs, and greenhouse governance: Knowledge, uncertainty, and international policy-making in a warming world. Applied Energy 86:258-264.

Miller, C. A. 2004. Climate science and the making of a global political order. In States of knowledge: The coproduction of science and social order, Hrsg. Sheila Jasanoff, 46-66. New York: Routledge.

Mumford, G. 2007. Psychology's ability to curb energy use. Monitor on Psychology 38 (11): $20-21$.

Najam, A., S. Huq, und Y. Sokona. 2003. Climate negotiations beyond Kyoto: Developing countries concerns and interests. Climate Policy 3:221-231.

Nelson, E., et al. 2008. Efficiency of incentives to jointly increase carbon sequestration and species conservation on a landscape. Proceedings of the National Academy of Sciences 105 (28): 9471-9476. 
Nelson, E., et al. 2009. Modeling multiple ecosystem services, biodiversity conservation, commodity production, and tradeoffs at landscape scales. Frontiers in Ecology and the Environment 7 (1): 4-11.

NRC (National Research Council). 2002. The drama of the commons. Committee on the Human Dimensions of Global Change, Elinor Ostrom, Thomas Dietz, Nives Dolšak, Paul Stern, Susan Stonich, und Elke Weber, Hrsg. Washington: National Academy Press.

Oppenheimer, M., B. C. O'Neill, und M. Webster. 2008. Negative learning. Climatic Change 89:155-172.

Ostrom, E. 1992. The rudiments of a theory of the origins, survival, and performance of commonproperty institutions. In Making the commons work: Theory, practice, and policy, Hrsg. Daniel W. Bromley et al., 293-318. San Francisco: ICS Press.

Ostrom, E. 2001. Reformulating the commons. In Protecting the commons: A framework for resource management in the Americas, Hrsg. Joanna Burger, Elinor Ostrom, Richard Norgaard, et al. 17-41. Washington: Island.

Ostrom, E., und H. Nagendra. 2006. Insights on linking forests, trees, and people from the air, on the ground, and in the laboratory. Proceedings of the National Academy of Sciences 103 (51): 19224-19231.

Ostrom, E., R. Gardner, und J. Walker. 1994. Rules, games, and common-pool resources. Ann Arbor: University of Michigan Press.

Posner, E. A., und C. Sunstein. 2008. Justice and climate change. Discussion Paper 08-04, Cambridge, MA, Harvard University Project on International Climate Agreements.

Poteete, A., M. Janssen, und E. Ostrom. 2010. Working together: Collective action, the commons, and multiple methods in practice. Princeton: Princeton University Press.

Pritchett, L., und M. Woolcock. 2003. Solutions when the solution is the problem: Arraying the disarray in development. World Development 35 (3): 435-461.

Rothstein, B. 1998. Just institutions matter: The moral and political logic of the universal welfare state. Cambridge: Cambridge University Press.

Rothstein, B. 2005. Social traps and the problem of trust. Cambridge: Cambridge University Press.

Sandler, T. 2004. Global collective action. Cambridge: Cambridge University Press.

Schultz, P., et al. 2007. The Constructive, destructive, and reconstructive power of social norms. Psychological Science 18 (5): 429-434.

Stavins, R. 1997. Policy instruments for climate change: How can national governments address a global problem? University of Chicago Legal Forum, vol. 1997: Rethinking Environmental Protection for the 21st Century, 293-329.

United Nations. 1982. Final act of the third conference on the law of the seas. Montenegro Bay: United Nations.

Vandenbergh, M. P., und A. C. Steinemann. 2007. The carbon-neutral individual. New York University Law Review 82 (December): 1673-1741.

Vandenbergh, M. P., J. Barkenbus, und J. Gilligan. 2008. Individual carbon emissions: The lowhanging fruit. UCLA Law Review 55:1701-1758.

Walters, C. J. 1986. Adaptive management of renewable resources. New York: Macmillan.

Wells, M., und K. Brandon. 1992. People and parks: Linking protected area management with local communities. Washington: World Bank.

Wiener, J. B. 2007. Think globally, act globally: The limits of local climate policies. University of Pennsylvania Law Review 155:1961-1979.

Wilson, J. 2002. Scientific uncertainty, complex systems, and the design of common-pool institutions. In The drama of the commons, Hrsg. National Research Council, Committee on the Human Dimensions of Global Change, Elinor Ostrom, Thomas Dietz, Nives Dolšak, et al., 327-359. Washington: National Academy Press. 